\title{
TEXTURE EFFECT IN X-RAY ANALYSIS OF RETAINED AUSTENITE IN STEELS
}

\author{
MATTI JÄRVINEN \\ Department of Information Technology, Lappeenranta University of Technology, \\ PO Box 20, SF-53851 Lappeenranta, Finland
}

(Received 24 October 1995; in final form 24 January 1996)

A method is described that can be used for determining the retained austenite concentration in steel samples that have texture. The method is based on symmetrized harmonics expansion for the representation of the orientation distribution of the crystallites in the sample and it presumes the use of specimen spinner on the data collection.

For demonstrating the application of this method integrated intensities of seven reflections from three steel samples with different degree of texture is measured using $C u K \alpha$ radiation and the retained austenite concentration is determined.

KEY WORDS: Quantitative analysis, X-ray diffraction, preferred orientation, texture effect.

\section{INTRODUCTION}

Steels are mainly mixtures of various polycrystalline iron phases whose crystal structure differs from each others. That is why diffraction methods suit very well for analysis of these materials. Direct comparison method is widely used for the measurement of the amount of retained austenite in hardened steels.

If the steel contains only austenite and ferrite phases, and the grains are randomly orientated, it is sufficient to measure carefully the integrated intensity of only one reflection from each phase, for the determination of true austenite concentration in the sample. However, the metallurgical samples have usually preferred orientation, or texture, that distorts the ideal intensity ratios and introduces erroneous value to the austenite content. In these cases more experimental information is needed to solve the problem.

If several pairs of reflections are measured, each pair can give a different value for the austenite concentration. The average value is often used as an estimate of the final outcome. In this paper is examined an other method that takes the special features of texture effect into account by an analytical texture model with adjustable parameters. The theoretical intensities are calculated by taking along this model, and the parameters, including the concentration parameters, are determined by fitting the theoretical intensities with the experimental data. 


\section{DESCRIPTION OF TEXTURE}

\subsection{Polar Axis Density}

Specimen rotation around an axis that is normal to the surface of the sample is commonly used for improving the particle statistics. The rotation axis, which is called here polar axis $\mathbf{P}$, defines in every crystallites of the sample a certain direction $(\vartheta, \varphi)$. The orientation distribution of the crystallites in the phase $q$ can be described by polar axis density $W_{q}$.

If $d V_{q}$ is the total volume of those crystallites of which the polar axis falls in a solid angle element $d \Omega$, in direction $(\vartheta, \varphi)$, (Figure 1 ), the polar axis density $W_{q}$ can be defined by the equation (Järvinen et al. 1970)

$$
W_{q}(\vartheta, \varphi)=\frac{d V_{q}}{V_{q}} / \frac{d \Omega}{4 \pi}
$$

where $V_{q}$ is the total volume of the illuminated crystallites in the phase $q$. For an ideal sample the orientation distribution is uniform so that $W_{i d} \equiv 1$

The measured integrated intensity of a reflection $h k l$ is proportional to the polar axis density in the direction of polar axis $\mathbf{P}$. In general, this direction can make an angle $\alpha$ with the scattering vector (Figure 2). The integrated intensity of a reflection from a spinning sample can be presented

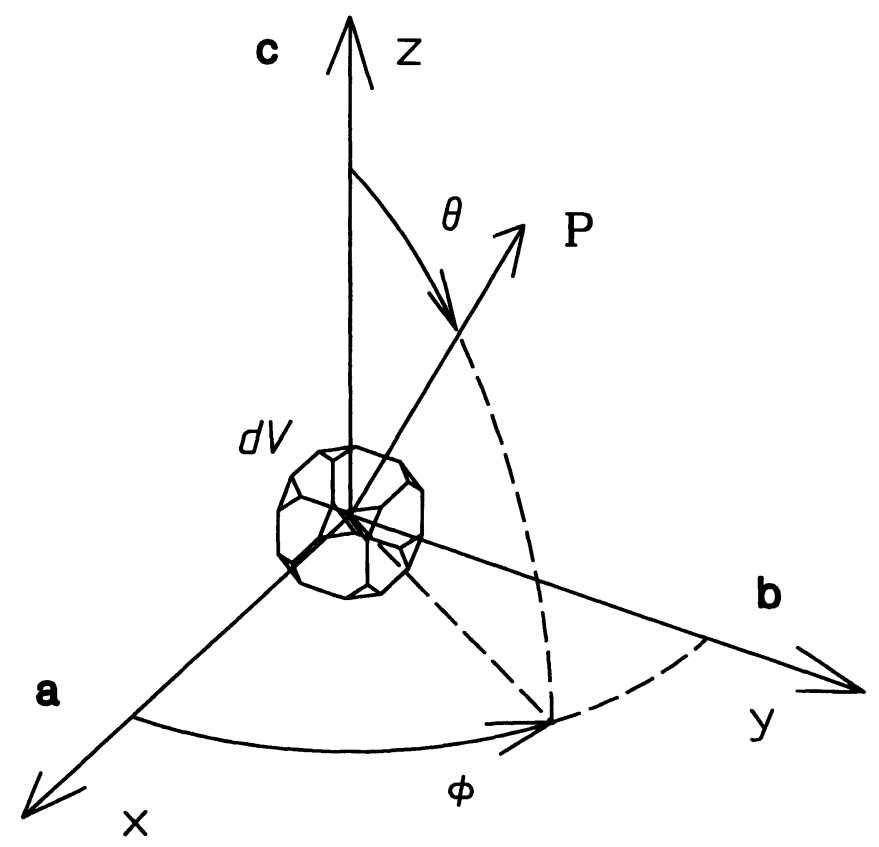

Figure 1 Direction of polar axis. The direction of the polar axis $\mathbf{P}$ is represented by the spherical coordinates $(\vartheta, \varphi)$ in the crystallographic coordinate system (abc) of a grain. In the cubic case the Cartesian coordinate system xyz coincides the crystallographic coordinate system. 


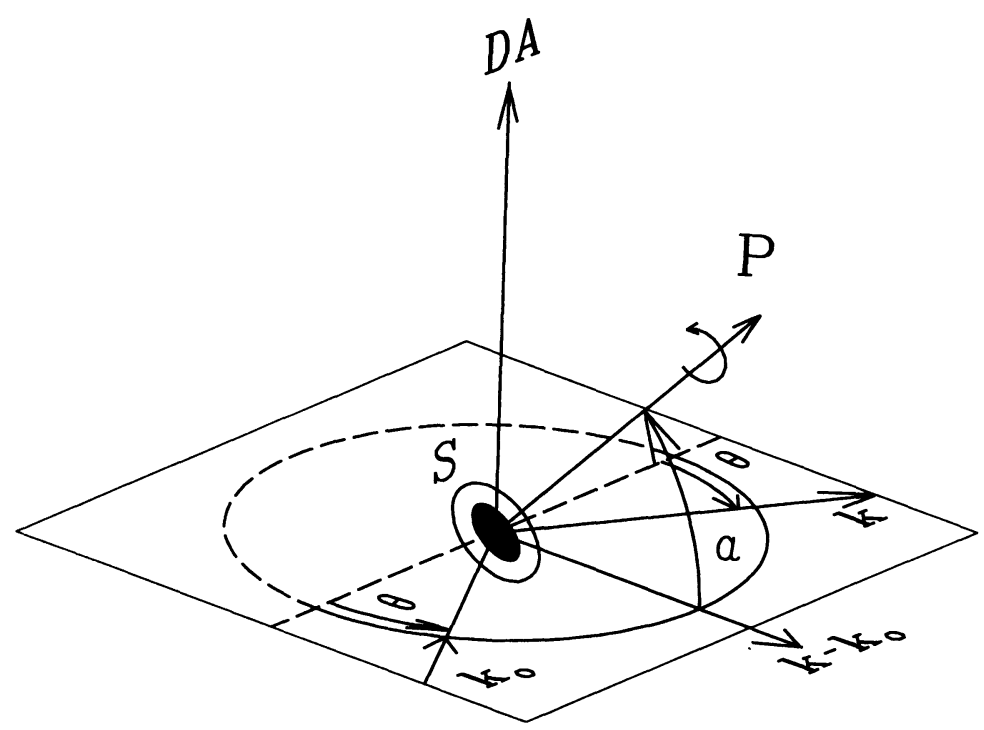

Figure 2 Diffraction geometry. The flat sample $S$ is rotating around the polar axis $P$. The vectors $k_{0}$ and $k$ are the directions of incident and diffracted beams, respectively, $k-k_{0}$ is the scattering vector. DA is the diffractometer axis and $\theta$ is the Bragg angle. The sample is inclined from the upright position at an angle $\alpha$.

$$
I_{o b}^{h k l}(\alpha)=T^{h k l} \bar{W}(h k l, \alpha)
$$

where

$$
\bar{W}(h k l, \alpha)=(1 / 2 \pi \sin \alpha) \oint_{O(h l k, \alpha)} W(\vartheta, \varphi) d s,
$$

The integration is performed along a circular path $O(h k l, \alpha)$ that is a circle on the surface of the unit sphere whose center is the pole $h k l$ and radius corresponds to the angle $\alpha$ (Figure 3). $T^{h k l}$ is a proportional constant whose value corresponds to the integrated intensity of an ideal sample and $\bar{W}$ is the average polar axis density on the circle $O$ that can also be called as texture factor of the sample (Bunge et al., 1989).

\subsection{Harmonic Model}

The polar axis density $W$ can be expanded in terms of a set of properly symmetrized harmonics $Y_{i j}$ (Järvinen 1993)

$$
W(\vartheta, \varphi)=\sum_{i, \text { even }}^{\infty} \sum_{j=1}^{N(i)} C_{i j} Y_{i j}(\vartheta, \varphi)
$$

where $C_{00}=1$ and the other $C_{i j}$ are adjustable coefficients. The index $i$ (that can get only even values) denotes the order of the harmonics and there are $N$ independent harmonics within each other.

The integration (3) yields

$$
\bar{W}(h k l, \alpha)=\sum_{i, j}^{2 M} C_{i j} Y_{i j}(h k l) P_{i}(\cos \alpha),
$$




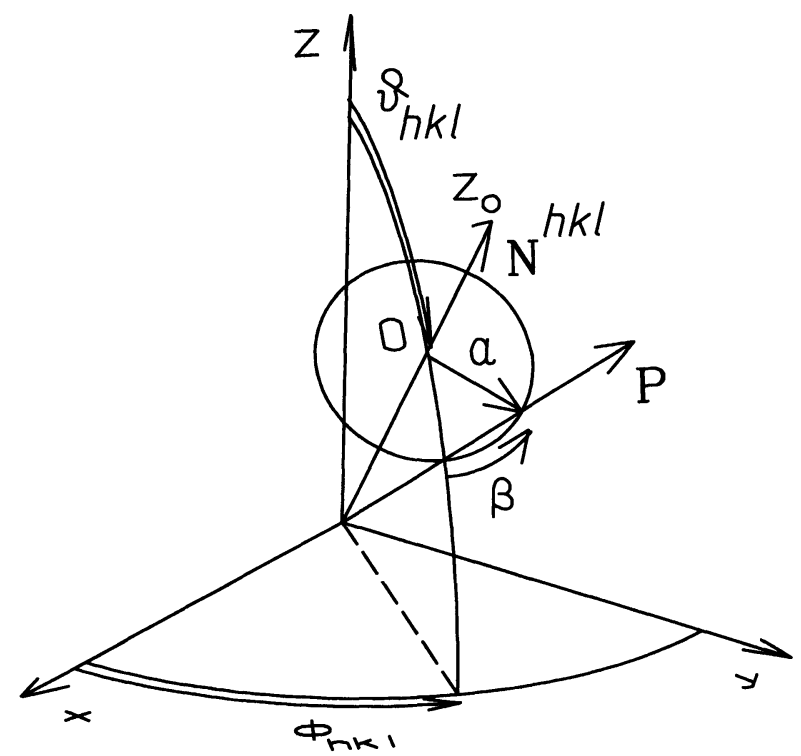

Figure 3 Orientation of the polar axis of the inclined sample in the grain coordinate system. The polar axes of diffracting grains are distributed on the circle whose center $O$ is in the direction of the plane normal $\mathbf{N}^{h k l}$ and whose radius corresponds to the inclination angle $\alpha$. The curves are on the surface of the unit sphere.

where $Y_{i j}(h k l)$ is the value of harmonics in the direction of the normal to the plane $h k l, P_{i}(\cos \alpha)=$ the Legendre polynomial and $P_{0} \equiv Y_{00} \equiv C_{00} \equiv 1$.

For practical purpose the series (5) is cut after the limit $i=2 M$ which is depending on the properties of the orientation distribution of the sample. Usually the harmonic expansion up to the 6th order is sufficient for reasonable accuracy in correction procedures.

\section{DETERMINATION OF AUSTENITE CONCENTRATION FROM SAMPLES WITH TEXTURE}

The diffracted intensity from the phase $q$ in the steel is given by

$$
I_{q}^{h k l}=w_{q} \frac{Q}{2 \mu} R_{q}^{h k l} \bar{W}_{q}(h k l, \alpha)
$$

where $Q$ is a common constant for all reflections including the fundamental electron quantities, wave-length, the power of primary beam and the radius of diffractometer circle. $\mu$ is the linear absorption coefficient of the steel that can be considered independent of the austenite concentration. $w_{q}$ is the volume fraction of the phase $q$ and $\mathrm{R}$ is a factor depending on the reflection $h k l$ and the phase $q$

$$
R_{q}^{h k l}=|F|^{2} p L(\theta) e^{-2 M} / v_{q}^{2}
$$


where $F=$ structure factor, $p=$ multiplicity factor, $L(\theta)=$ Lorenz-polarization factor, $\theta=$ the Bragg angle, $e^{-2 M}=$ temperature factor, $v=$ volume of unit cell.

If for brevity is written

$$
w_{q} Q / 2 \mu=x_{q}
$$

and

one gets from (6)

$$
I_{q}^{h k l} / R_{q}^{h k l}=G_{q}^{h k l}
$$

$$
G_{q}^{h k l}=x_{q} \bar{W}_{q}(h k l, \alpha)
$$

for each reflection $h k l$ and each phase $q$. From these equations it can be seen that $x_{q}$ is a quantity that is closely related to the volume fraction $w_{q}$ and in the absence of preferred orientation (when $\bar{W}_{q}=1$ ) its value should be the same as the value of the intensity proportion $G_{q}$.

Ferrite and austenite are both cubic crystals so that in series expansion (5) up to 6 th order $(M=3)$ there are only two terms that vary with angles (Table 1 . The cubic harmonics are denoted by $K^{i}$ ). In intensity measurements the symmetrical reflection method was used so that the polar angle $\alpha=0$ and, hence, all the Legendre polynomials $P_{i} \equiv 1$. Thus the texture factors gets the form

$$
\bar{W}_{q}(h k l)=1+C_{q}^{4} K^{4}(h k l)+C_{q}^{6} K^{6}(h k l)
$$

When several reflections from both phases are measured the ratios $x_{q}$ can be solved from the following set of equations (subscript a for austenite and $f$ for ferrite)

$$
\begin{array}{cc}
x_{a}+x_{a} C_{a}^{4} K^{4}(h k l)+x_{a} C_{a}^{6} K^{6}(h k l) & =G_{a}^{h k l} \\
\vdots & =\quad \vdots \\
x_{f}+x_{f} C_{f}^{4} K^{4}(h k l)+x_{f} C_{f}^{6} K^{6}(h k l) & =G_{f}^{h k l} \\
\vdots & =\quad
\end{array}
$$

The values of $K^{i}(h k l)$ are given in Table 2.

If more than three reflections are measured from both phases, least squares fitting can be used for calculating the unknown parameters. Type $x C$ product can be treated as a single parameter in the fitting procedure.

Table 1 Cubic harmonics $K^{i}(\vartheta, \varphi), M=3$.

\begin{tabular}{l}
$x=\sin \vartheta \cos \varphi, y=\sin \vartheta \cos \varphi, z=\cos \vartheta$ \\
\hline$K^{0}=1$ \\
$K^{4}=\frac{5}{2}\left(x^{4}+y^{4}+z^{4}-\frac{3}{5}\right)$ \\
$K^{6}=\frac{693}{32}\left(x^{6}+y^{6}+z^{6}-\frac{6}{11} K^{4}-\frac{3}{7}\right)$
\end{tabular}


Table 2 Values of cubic harmonics for different reflections.

\begin{tabular}{lrrrr}
\hline$h k l$ & $\vartheta^{\circ}$ & $\varphi^{\circ}$ & \multicolumn{1}{c}{$K^{4}$} & \multicolumn{1}{c}{$K^{\circ}$} \\
\hline Ferrite & & & & \\
200 & 0.0 & 0.0 & 1.000 & 0.563 \\
211 & 35.3 & 45.0 & -0.250 & 0.289 \\
220 & 45.0 & 0.0 & -0.250 & -0.914 \\
222 & 54.7 & 45.0 & -0.667 & 1.000 \\
Austenite & & & & \\
200 & 0.0 & 0.0 & 1.000 & 0.563 \\
220 & 45.0 & 0.0 & -0.250 & -0.914 \\
311 & 25.2 & 45.0 & 0.215 & 0.074 \\
222 & 54.7 & 45.0 & -0.667 & 1.000 \\
\hline
\end{tabular}

Supposing that the steel contains only ferrite and austenite an additional equation can be written

$$
w_{a}+w_{f}=1
$$

Further, taking the equation (8) into account, it can be seen that

$$
x_{a}+x_{f}=Q / 2 \mu
$$

so that for the volume concentration of austenite will be obtained the expression

$$
w_{a}=\frac{x_{a}}{x_{a}+x_{f}}
$$

\section{EXPERIMENTAL}

X-ray diffraction measurements were made with a standard, computer controlled diffractometer using symmetrical reflection method. The samples were cut from a laser beam welded, austenitic-ferritic dissimilar steel joints. The intensity from an angular range of $48-100^{\circ}$ in $2 \theta$ was collected in steps of $0.02^{\circ}$ for $2 \mathrm{~s}$ using monochromatized $C u K \alpha$ radiation. The diameter of the incident beam was about $1 \mathrm{~mm}$ at the sample site. Diffraction profiles from three samples (a), (b) and (c), with different amount of austenite content, are seen in the Figure 4.

From the measured profiles the integrated intensities of reflections $200,220,311$, 222 were calculated for austenite and 200,211, 220 for ferrite. The results are given in Table 3.

\section{RESULTS AND DISCUSSION}

The results of the harmonic analysis are given in Table 4. It can be seen that the degree of texture of different samples and of different phases vary considerably. In the sample (a) the texture is strongest, the value of texture factor $\bar{W}$ varies from 0.58 to 1.58 . If for this sample the amount of retained austenite is calculated from a single pair of reflections the outcomes would vary from $8 \%$ to $23 \%$ and average result would be $14.9 \%$. The analysis with the harmonics model gives the answer $8.8 \%$. 


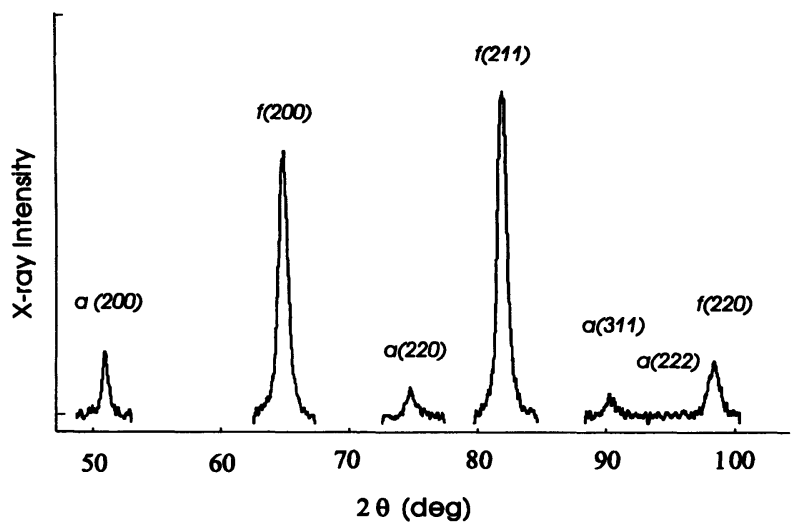

(a)

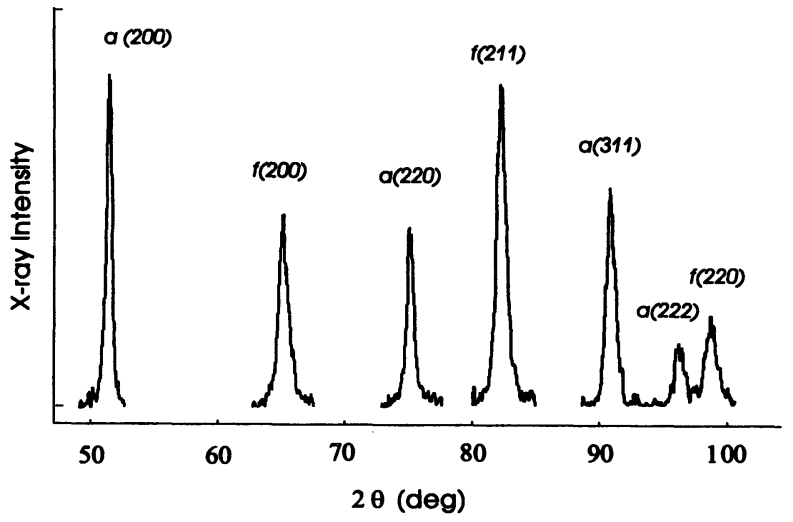

(b)

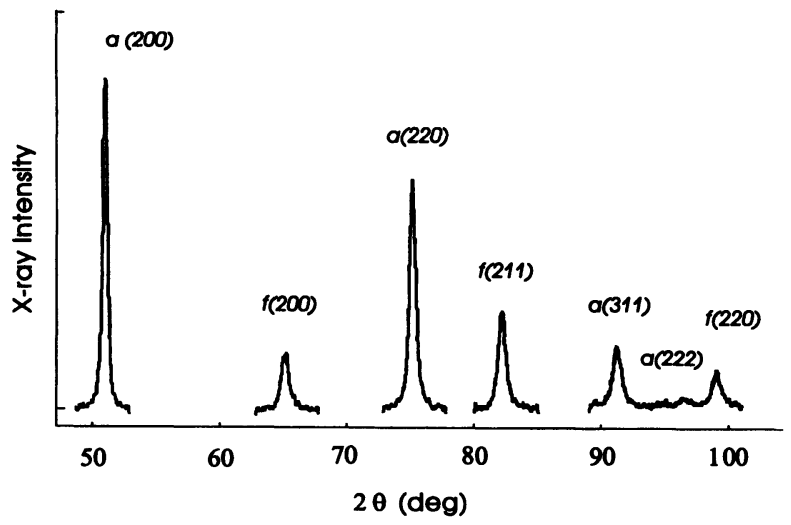

(c)

Figure $4 \mathrm{X}$-ray diffraction profiles from the three steel samples $\mathrm{a}, \mathrm{b}$ and $\mathrm{c}$, with different degree of texture and with different amount of austenite content. 
Table 3 Theoretical and measured integrated intensities of samples a, b and c, for different reflections.

\begin{tabular}{lrccc}
\hline$h k l$ & $R$ & $I_{a}$ & $I_{b}$ & $I_{c}$ \\
\hline Ferrite & & & & \\
200 & 69.0 & 50.4 & 28.1 & 18.8 \\
211 & 130.9 & 61.7 & 44.1 & 29.7 \\
220 & 44.4 & 11.8 & 14.7 & 12.5 \\
222 & 36.1 & $18.4^{*}$ & $9.6^{*}$ & $6.9^{*}$ \\
Austenite & & & & \\
200 & 156.5 & 9.8 & 32.8 & 63.7 \\
220 & 87.7 & 5.6 & 21.0 & 57.8 \\
311 & 105.3 & 4.4 & 28.8 & 20.8 \\
222 & 31.6 & 0.1 & 7.9 & 2.2 \\
\hline
\end{tabular}

*determined by iteration

Table 4 Results of harmonic analysis. $d e v$ is the deviation of measured intensities from the theoretical values in percentages, RA is the amount of retained austenite in the sample.

\begin{tabular}{|c|c|c|c|c|c|}
\hline \multicolumn{6}{|l|}{ Sample a. } \\
\hline$h k l$ & $G \%$ & $\bar{W}$ & dev\% & $C_{4}$ & $C_{6}$ \\
\hline $\begin{array}{c}\text { Ferrite } \\
200 \\
211 \\
220 \\
222\end{array}$ & $\begin{array}{r}x_{f}=46.3 \\
73.0 \\
47.1 \\
26.6 \\
51.0\end{array}$ & $\begin{array}{l}1.58 \\
1.01 \\
0.58 \\
1.11\end{array}$ & $\begin{array}{r}-0.1 \\
0.4 \\
-0.1 \\
-0.2\end{array}$ & 0.38 & 0.36 \\
\hline $\begin{array}{l}\text { Austenite } \\
200 \\
220 \\
311 \\
222\end{array}$ & $\begin{array}{r}x_{a}=4.5 \\
6.2 \\
6.4 \\
4.8 \\
0.1\end{array}$ & $\begin{array}{r}1.32 \\
1.37 \\
1.10 \\
-0.01\end{array}$ & $\begin{array}{r}0.3 \\
0.3 \\
-0.7 \\
0.1\end{array}$ & 0.64 & -0.58 \\
\hline
\end{tabular}

Sample b.

\begin{tabular}{lrllll}
\hline$h k l$ & $G \%$ & $\bar{W}$ & $\operatorname{dev} \%$ & $C_{4}$ & $C_{6}$ \\
\hline Ferrite & $x_{f}=34.2$ & & & 0.22 & -0.04 \\
200 & 40.7 & 1.20 & -0.3 & & \\
211 & 33.7 & 0.93 & 1.8 & & \\
220 & 33.1 & 0.99 & -0.6 & & \\
222 & 26.7 & 0.81 & -0.9 & & -0.01 \\
Austenite & $x_{a}=24.5$ & & & \\
200 & 20.9 & 0.91 & -1.4 & & \\
220 & 23.9 & 1.03 & -1.3 & & \\
311 & 27.3 & 0.98 & 3.3 & & \\
222 & 25.0 & 1.05 & 0.6 & & \\
\hline \multicolumn{5}{c}{ Mean dev. $=1.6, R A=41.7 \%$} \\
\hline
\end{tabular}


Table 4 Cont'd.

\begin{tabular}{|c|c|c|c|c|c|}
\hline \multicolumn{6}{|l|}{ Sample c. } \\
\hline$h k l$ & $G \%$ & $\bar{W}$ & dev\% & $C_{4}$ & $C_{6}$ \\
\hline Ferrite & $x_{f}=25.3$ & & & 0.16 & -0.16 \\
\hline 200 & 27.2 & 1.07 & 0.1 & & \\
\hline 211 & 22.7 & 0.92 & -0.5 & & \\
\hline 220 & 28.1 & 1.10 & 0.2 & & \\
\hline 222 & 19.0 & 0.74 & 0.3 & & \\
\hline Austenite & $x_{a}=43.8$ & & & 0.30 & -0.64 \\
\hline 200 & $\begin{array}{ll}a & 40.7\end{array}$ & 0.94 & -0.2 & & \\
\hline 220 & 65.9 & 1.51 & -0.2 & & \\
\hline 311 & 45.0 & 1.02 & 0.5 & & \\
\hline 222 & 7.1 & 0.16 & -0.1 & & \\
\hline \multicolumn{6}{|c|}{ Mean dev. $=0.3, R A=63.3 \%$} \\
\hline
\end{tabular}

In the sample (b) the texture is lighter, especially in the austenite phase. In this case the retained austenite values, calculated from single pairs of reflections, varies from $33 \%$ to $52 \%$ and the average value $42.2 \%$ is very close to the value $41.7 \%$, which is calculated using the harmonics model. In the case of sample (c), the texture is again stronger. The single pair values vary from $42 \%$ to $75 \%$, the average value being $58.3 \%$. The harmonic analysis gives the result $63.3 \%$.

The first harmonic analyses were made by using only three reflections per phase, but the results seemed to be unreliable. If the 4th reflection is taken into the analyses the agreement between the measured and the calculated intensities is much better. Unfortunately, it was not possible to measure the 222 reflection for the austenite phase, so that these values were calculated by iteration using the rest of the intensity data.

It can be seen that utilizing the average value of several pairs of reflections give much more reliable and accurate outcomes than the single pair measurements. However, when several reflections are measured, already a modest texture model puts the analysis on firmer bases and improves the accuracy of the result and in addition gives information about the texture of the sample.

The cubic harmonics model suits particularly well the analysis of retained austenite in steels. The good agreement between the measured and the theoretical intensity values indicates that the results for the calculated amount of retained austenite are reliable and quite accurate, the error due to the texture is clearly less than $1 \%$. If more accurate results about retained austenite concentration are needed the intensity measurements must be done more carefully and the other systematical errors to be studied. More experimental information about the texture of the sample can be obtained by variating the $\alpha$ angle by making unsymmetrical reflection measurements or by inclining the sample from the upright position.

\section{References}

Bung, H. J., Dahms, M. and Brokmeier, H. G. (1989). The Determination of Integrated Intensities from Polycrystalline Samples with Preferred Orientation. Cryst. Rev., Vol. 2. pp. 67-88.

Järvinen, M. (1993). Application of Symmetrized Harmonics Expansion to Correction of the Preferred Orientation Effect, J. Appl. Cryst., 26, 525-531.

Järvinen, M., Merisalo, M., Pesonen, A. and Inkinen, O. (1970). Correction of Integrated X-ray Intensities for Preferred Orientation in Cubic Powders, J. Appl. Phys., 3, 313-318. 